\title{
Cordycepin induces apoptosis of human acute monocytic leukemia cells via downregulation of the ERK/Akt signaling pathway
}

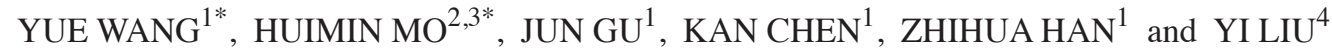 \\ ${ }^{1}$ Department of Cardiology, Ninth People's Hospital, Shanghai Jiaotong University Medical \\ School, Shanghai 200011; ${ }^{2}$ Institute of Hematology, Xuzhou Medical University; ${ }^{3}$ Department of Hematology, \\ The Affiliated Hospital of Xuzhou Medical University, Xuzhou, Jiangsu 221002; ${ }^{4}$ Department of Ultrasound, \\ Renji Hospital, Shanghai Jiaotong University Medical School, Shanghai 200127, P.R. China
}

Received August 29, 2016; Accepted May 19, 2017

DOI: $10.3892 / \mathrm{etm} .2017 .4855$

\begin{abstract}
The aim of the present study was to examine the apoptotic effect of cordycepin (COR) on human THP-1 acute monocytic leukemia cells. THP-1 cells were exposed to different concentrations of COR for 24, 48, 72 or $96 \mathrm{~h}$. The cell viability and apoptotic rate were analyzed. The gene expression of Akt1, Akt2, Akt3, B-cell lymphoma 2 (Bcl-2) and Bcl-2-associated $\mathrm{X}$ protein (Bax) were assessed by reverse-transcription quantitative PCR. Western blot analysis was used to detect the protein levels of phosphorylated (p)-Akt, p-extracellular signal-regulated kinase (ERK) and cleaved caspase-3. It was found that the viability of THP-1 cells was inhibited by COR in a dose- and time-dependent manner. After treatment with $200 \mu \mathrm{M}$ COR for $24 \mathrm{~h}$, the percentage of apoptotic cells was significantly increased. COR also downregulated the levels of Bcl-2, Akt1, Akt2 and Akt3, and elevated the expression of Bax. The protein levels of p-Akt and p-ERK were suppressed and cleaved caspase-3 was increased after treatment of COR. In conclusion, COR was found to induce apoptosis of THP-1 acute monocytic leukemia cells through downregulation of ERK/Akt signaling.
\end{abstract}

\section{Introduction}

Acute myeloid leukemia (AML) is a clonal disorder, which comprises a group of clonal malignant diseases. The leukemia

Correspondence to: Dr Yi Liu, Department of Ultrasound, Renji Hospital, Shanghai Jiaotong University Medical School, 160 Pujian Road, Shanghai 200127, P.R. China

E-mail: doctorliuyi@126.com

Dr Zhihua Han, Department of Cardiology, Ninth People's Hospital, Shanghai Jiaotong University Medical School, 639 Zhizaoju Road, Shanghai 200011, P.R. China

E-mail: doctorhanzhihua@163.com

*Contributed equally

Key words: cordycepin, THP-1, apoptosis, ERK, Akt, caspase-3 cells originate from the bone marrow, giving rise to an accumulation of abnormal immature myeloid cells in the bone marrow and blood $(1,2)$. AML is one of the most common leukemia types. Over $50 \%$ of patients diagnosed with AML are $>65$ years of age (3). The main therapeutic strategies for AML patients are aggressive chemotherapeutic regimens and hematopioetic stem cell transplantation, and $\sim 40 \%$ of AML patients receive chemotherapy within 3 months after diagnosis $(4,5)$. In spite of intensive chemotherapy being able to achieve complete remission in most AML patients, the overall survival rate remains poor and the therapeutic process is usually associated with serious adverse events (6). However, AML comprises the following 8 types: $\mathrm{M}_{0}$, minimally differentiated AML; $\mathbf{M}_{1}$, AML without maturation; $\mathrm{M}_{2}$, AML with maturation; $\mathrm{M}_{3}$, acute pro-myelocytic leukemia; $\mathrm{M}_{4}$, acute myelomonocytic leukemia; $\mathrm{M}_{5}$, acute monocytic leukemia (AMoL); $\mathrm{M}_{6}$, erythroleukemia; and $\mathrm{M}_{7}$, acute megakaryoblastic leukemia. And there is no treatment plan for any of the AML typse (7-10). The major objective of the present study was to identify a drug for curing AMoL.

The development of AMoL is a multistep and multifactorial process. The neoplastic cells are most frequently derived from white blood cells, have a high rate of marrow infiltration and high bad karyotype ratio. As AMoL is insensitive to numerous chemotherapy regimens, affected patients have a low remission rate and short lifetime (11-16). Therefore, a novel and effective drug is urgently required.

In recent years, bioactive natural products have emerged and received a considerable amount of attention from researchers. Cordyceps militaris is a traditional Chinese medicinal plant, which has been widely used for a long time. Recent studies have demonstrated that biologically active components isolated from Cordyceps species have various pharmacological effects $(17,18)$. Cordycepin (COR) is one of the most widely studied active components of Cordyceps militaris and has diverse biological functions, such as anti-tumor (19), anti-invasive (20) and anti-inflammatory effects (21). However, the effects and potential mechanisms of COR in AMoL have largely remained to be elucidated. Therefore, the purpose of the present study was to evaluate the anti-cancer effect of COR on AMoL and investigate the potential underlying mechanisms. The present findings suggested that COR induces apoptosis of the THP-1 AMoL cell line via deactivating Akt and 
extracellular signal-regulated kinase (ERK) and upregulating the expression of cleaved caspase-3 indicating that COR may be a potential therapeutic drug for AMoL.

\section{Materials and methods}

Reagents. COR and phorbol-12-myristate-13-acetate (PMA) were purchased from Sigma-Aldrich (Merck KGaA, Darmstadt, Germany). COR was dissolved in dimethyl sulfoxide (DMSO; Sigma-Aldrich; Merck KGaA), and the proportion of DMSO in the culture medium was $<0.05 \%$. COR was stored at $-20^{\circ} \mathrm{C}$. Primary antibodies against phosphorylated (p)-ERK (Cat no. 4348S), total (t)-ERK (Cat no. 4695S), p-Akt (Cat no. 4060S), t-Akt (Cat no. 4685S), cleaved caspase-3 (Cat no. 1050S), $\beta$-actin (Cat no. 4970S) as well as Anti-rabbit Immunoglobulin $\mathrm{G}(\mathrm{IgG})$ secondary antibodies (Cat no. 5151S) were purchased from Cell Signaling Technology, Inc. (Danvers, MA, USA).

Cell culture. THP-1 AMoL cells were purchased from the Cell Bank of the Chinese Academy of Sciences (Shanghai, China). THP-1 cells were cultured in RPMI 1640 medium (Hyclone; GE Healthcare, Little Chalfont, UK) containing $10 \%$ fetal bovine serum (Hyclone; GE Healthcare), $100 \mathrm{U} / \mathrm{ml}$ penicillin and $100 \mu \mathrm{g} / \mathrm{ml}$ streptomycin (Hyclone; GE Healthcare) in an incubator at $37^{\circ} \mathrm{C}$ with $5 \% \mathrm{CO}_{2}$. All cells we used at a passage of $<20$.

Measurement of cell viability. A Cell Counting Kit-8 (CCK-8; Dojindo, Kumamoto, Japan) was used to assess the potential therapeutic effect of COR. For measurement of cell proliferation, THP-1 AMoL cells were seeded into each well of a 96-well plate at a density of 20,000 cells/well in $100 \mu 1$ culture media containing $100 \mathrm{nM}$ PMA to induce adherence, and treated with $0,25,50,100,150,200 \mu \mathrm{mol} / 1 \mathrm{COR}$ for $96 \mathrm{~h}$ after adherence (22). Following treatment with COR for 24, 48, 72 and 96 h, $100 \mu \mathrm{l}$ culture media and $10 \mu \mathrm{l}$ CCK-8 solution were added to each well, followed by incubation at $37^{\circ} \mathrm{C}$ for $150 \mathrm{~min}$. The optical density (OD) value at $450 \mathrm{~nm}$ was determined using a microplate reader (BioTek Instruments, Inc., Winooski, VT, USA). Each experimental condition was repeated in three wells. The cell viability relative to that in the $0 \mu \mathrm{mol} / 1 \mathrm{COR}$ group (control) was calculated using the following equation: Cell viability (\% of control group $)=\mathrm{OD}_{\text {drug-treated group }} / \mathrm{OD}_{\text {control group }}$.

Apoptosis assay. THP-1 cells were incubated with various concentrations of COR for $24 \mathrm{~h}$. Subsequently, the cells were washed twice with cold PBS and harvested. The cells were re-suspended in 1X Annexin-binding buffer, and stained with Annexin V-fluorescein isothiocyanate (FITC) and propidium iodide (PI; BD Biosciences, Franklin Lakes, NJ, USA). The apoptotic rate was measured by flow cytometry using a BD FACSCalibur $^{\mathrm{TM}}$ analyzer (BD Biosciences, Franklin Lakes, NJ, USA). The total apoptotic rate of cells was considered to be the early apoptotic rate (lower right quadrant in the PI vs. FITC dot plot) plus the late apoptotic rate (upper right quadrant in the dot plot).

$R N A$ isolation and reverse-transcription quantitative polymerase chain reaction ( $R T-q P C R)$. THP-1 cells were cultured in complete medium in the presence of various concentrations of COR for $24 \mathrm{~h}$. The total RNA of the THP-1 cells was then isolated using TRIzol reagent (Invitrogen; Thermo Fisher Scientific, Inc., Waltham, MA, USA) following the manufacturer's instructions. For first-strand complementary DNA (cDNA) synthesis, RT was performed using the 1st Strand cDNA Synthesis kit (Takara Bio Inc., Dalian, China). The relative gene expression was determined by real-time PCR using the SYBR Premix Ex Taq kit (Takara Bio, Inc.) with the ABI Prism 7500 Fast Real-Time PCR system (Applied Biosystems; Thermo Fisher Scientific, Inc.). The real-time PCR thermocycling conditions were as follows: pre-degeneration at $95^{\circ} \mathrm{C}$ for $30 \mathrm{sec}$, followed by 40 cycles at $95^{\circ} \mathrm{C}$ for $5 \mathrm{sec}$ and $60^{\circ} \mathrm{C}$ for $34 \mathrm{sec}$, and the dissociation stage was $34 \mathrm{sec}$ at $95^{\circ} \mathrm{C}$. The primers were designed and selected using the Primer-BLAST tool (https://www.ncbi.nlm.nih. gov/tools/primer-blast/). Gene expression was quantified using the $2^{-\Delta \Delta C q}$ method (23). $\beta$-actin was used as the internal control. The primer sequences are listed in Table I.

Western blot analysis. THP-1 cells were treated as for the RT-qPCR analysis and then lysed with radioimmunoprecipitation assay buffer (Upstate Biotechnology, Inc., Lake Placid, NY, USA) supplemented with a protease inhibitor cocktail (Roche Diagnostics GmbH, Mannheim, Germany). For western blot analysis, protein concentration was quantified using the BCA Protein Assay kit (Thermo Fisher Scientific, Inc.; catalogue no. 23250) according to manufacturer protocol. Samples were adjusted to the same protein concentration prior to loading (30 $\mu \mathrm{g}$ each sample) and separated by $10 \%$ SDS-PAGE for p-Akt, t-Akt, p-ERK, t-ERK and $\beta$-actin and $12.5 \%$ SDS-PAGE for cleaved caspase-3. Subsequently, proteins were transferred onto a nitrocellulose membrane (Merck Millipore, Billerica, MA, USA). Antibodies against p-Akt, t-Akt, p-ERK, t-ERK and cleaved caspase- 3 were applied at the dilutions recommended by the manufacturer $(1: 1,000)$ at $4^{\circ} \mathrm{C}$ overnight. $\beta$-actin $(1: 1,000)$ was used as a loading control. After $37 \mathrm{~min}$ washes in Tris-buffered saline containing Tween-20 (TBST), the membranes were incubated with anti-rabbit second antibody (1:15,000; Cell Signalling Technology, Inc) for $1 \mathrm{~h}$ at room temperature. The target proteins were detected using the Odyssey Infrared Imaging System (Li-Cor Biosciences, Lincoln, NE, USA). The quantification of the bands was analyzed by the software of Odyssey Infrared Imaging System (Version 3.0).

Statistical analysis. All of the experiments were repeated 3 times. Values are expressed as the mean \pm standard deviation. Statistical analysis was performed by one-way analysis of variance, followed by Duncan's post-hoc test using SPSS 19.0 (International Business Machines, Corp., Armonk, NY, USA). $\mathrm{P}<0.05$ was considered to indicate a statistically significant difference.

\section{Results}

COR inhibits THP-1 cell proliferation. To assess the effects of COR on the proliferation of THP-1 cells, a CCK-8 assay using was performed. The inhibitory effect of COR on the proliferation of THP-1 cells was strengthened with 
Table I. Sequences of primers used for polymerase chain reaction.

\begin{tabular}{lll}
\hline Gene & Direction & \multicolumn{1}{c}{ Primer sequence (5'-3') } \\
\hline Akt1 & Forward & GAAGGACGGGAGCAGGC \\
& Reverse & CTCACGCGCTCCTCTCAG \\
Akt2 & Forward & TGCCACCATGAATGAGGTGAA \\
& Reverse & GTACCCAATGAAGGAGCCGT \\
Akt3 & Forward & TTTCTCCAAGTTGGGGGCTC \\
& Reverse & CCCCTCTTCTGAACCCAACC \\
Bcl-2 & Forward & TTTGTGGAACTGTACGGCCC \\
& Reverse & GTTGACTTCACTTGTGGCCC \\
Bax & Forward & AGCAGATCATGAAGACAGGGG \\
& Reverse & TGCTCGATCCTGGATGAAACC \\
$\beta$-actin & Forward & CTCACCATGGATGATGATATCGC \\
& Reverse & AGGAATCCTTCTGACCCATGC \\
\end{tabular}

Bcl-2, B-cell lymphoma 2; Bax, Bcl-2-associated X protein.

the increase of the COR concentration after 24, 48, 72 and $96 \mathrm{~h}$ of treatment (Fig. 1). In conclusion, COR inhibited the proliferation of THP-1 cells in a dose- and time-dependent manner.

COR concentration-dependently induces apoptosis in THP-1 cells. As presented in Fig. 2, flow cytometric analysis was employed to detect COR-induced apoptosis. Different concentrations of COR increased the total apoptotic rate in a dose-dependent manner $(3.35 \%$ in the control vs. 11.48 , 13.67 and $24.99 \%$ after treatment with COR at 100,150 and $200 \mu \mathrm{M}$, respectively; $\mathrm{P}<0.05$; Fig. 2A-G). Quantitative results on the early and late apoptotic rates were consistent with this phenomenon (Fig. 2H-I). To conclude, COR was found to exert its inhibitory function on THP-1 cells by induction of apoptosis, resulting in cell death.

COR regulates apoptosis- and survival-associated gene expression in THP-1 cells. To investigate the in-depth molecular mechanisms of the inhibitory effect of COR on THP-1 human monocytic leukemia cells, RT-qPCR was performed to examine its impact on the expression of apoptosis-associated genes. It was found that the expression levels of Bcl-2 were reduced in COR-treated THP-1 cells in a dose-dependent manner, while the expression of Bax was upregulated (Fig. 3). In addition, Akt signaling is crucial for the initiation and progression of AML and also regulates a number of downstream targets responsible for cell survival and proliferation $(24,25)$. Therefore, the current study detected the mRNA level of three isoforms (Akt1, Akt2 and Akt3) of Akt. The cell survival-associated genes Akt1, Akt2 and Akt3 were downregulated after COR treatment (Fig. 4). Therefore, the phosphorylation of Akt was detected by western blot analysis based on the levels of mRNA (Fig. 4). These results indicated that these genes are potentially involved in signaling pathways downstream of those targeted by COR in monocytic leukemia cells.
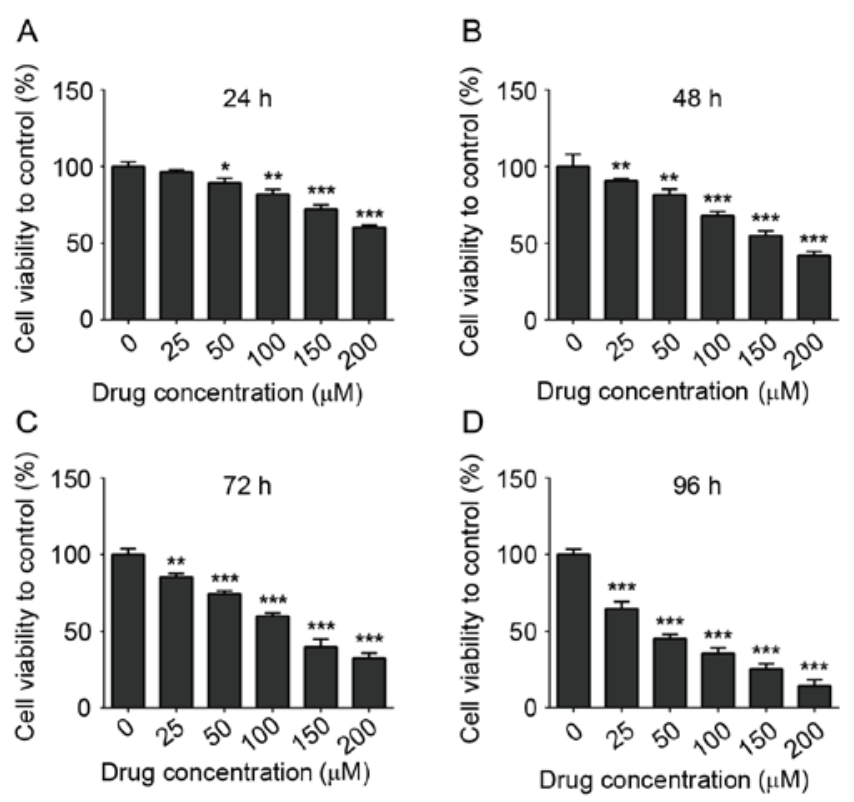

Figure 1. Anti-proliferative effects of COR on THP-1 cells. (A-D) A Cell Counting Kit- 8 assay demonstrated the anti-proliferative effects of COR on THP-1 cells after treatment for 24, 48, 72 and $96 \mathrm{~h}$. Values are expressed as the mean \pm standard error. ${ }^{*} \mathrm{P}<0.05 ;{ }^{* *} \mathrm{P}<0.01 ;{ }^{* * *} \mathrm{P}<0.001$ vs. untreated group. COR, cordycepin.

Akt and ERK are potential downstream targets of COR. To evaluate the potential involvement of the signal transduction pathways and the mechanisms of the effects of COR on THP-1 cells, the expression of various signaling proteins was determined in THP-1 cells by western blot analysis following treatment with different concentrations of COR. The results demonstrated that COR inhibited the protein expression of p-Akt and p-ERK in a concentration-dependent manner significantly $(\mathrm{P}<0.05$; Fig. 5A-D). However, cleaved caspase-3 was significantly activated by increasing concentrations of COR $(\mathrm{P}<0.05$; Fig. 5E and F). Taken together, these results suggested that Akt and ERK are potential downstream targets of COR in THP-1 cells, and that apoptosis is induced via associated signaling pathways.

\section{Discussion}

The major challenge in the treatment of AML is the high failure and relapse rate due to drug resistance. Application of novel drug treatments is one of the therapeutic approaches for patients with resistance to standard therapies. Clinical evaluation of potential effective chemotherapeutic drugs may provide novel treatments, which may improve the prospects of refractory leukemia patients. AMoL is a rare but distinct disease entity, which is most remarkable due to its clinical course. Cytogenetic characterization is essential for the diagnosis and determination of the prognosis of patients (26-28). There is an urgent requirement to identify less toxic and more effective treatments, and an increasing amount of research has focused on the application of natural products in $\mathrm{AMoL}$ treatment.

COR was previously reported to have anti-tumor $(29,30)$, anti-inflammatory (31) and anti-oxidant (32) activities. However, the potential role and mechanisms of COR in the treatment of AMoL have remained to be determined. The 
A

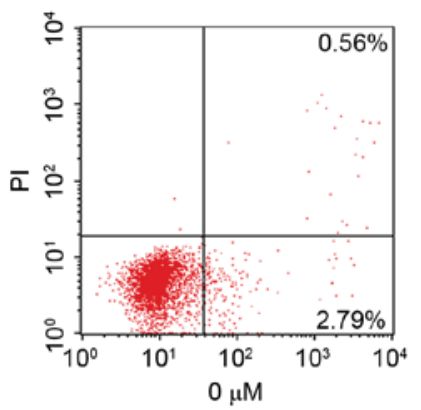

D

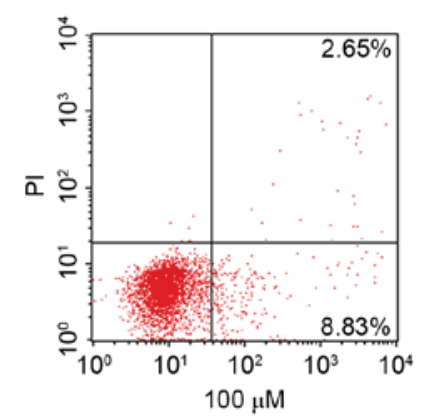

G

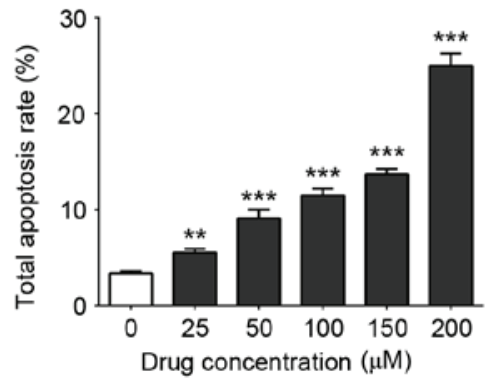

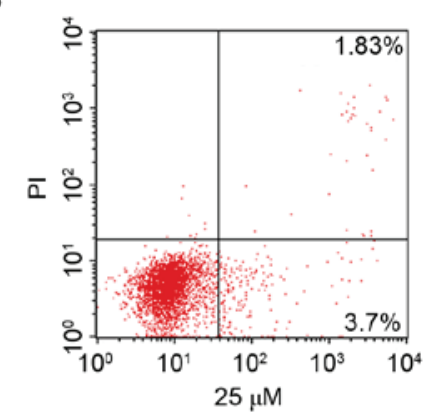

$\mathrm{E}$

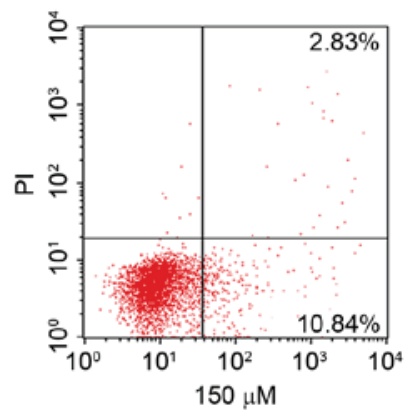

$\mathrm{H}$

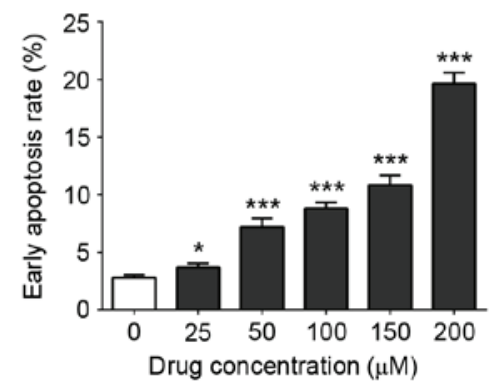

C

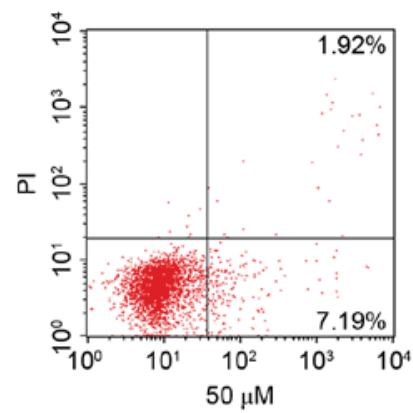

$\mathrm{F}$
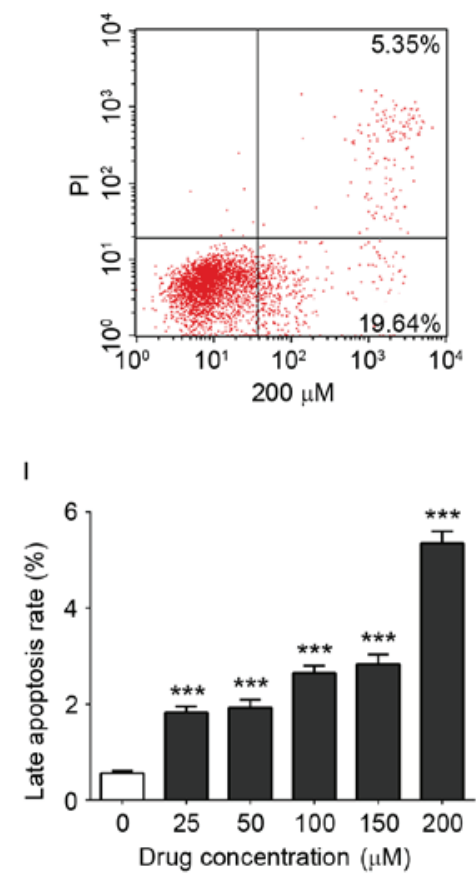

Figure 2. Apoptosis of THP-1 cells following COR treatment. (A-F) Flow cytometry-based assessment of apoptosis in THP-1 cells following treatment with $0,25,50,100,150,200 \mu \mathrm{M}$ of COR. Annexin V-fluorescein isothiocyanate is displayed on the x-axis. (G-I) Quantitative analysis of total, early and late apoptotic rate. Values are expressed as the mean \pm standard error. ${ }^{*} \mathrm{P}<0.05 ;{ }^{* * *} \mathrm{P}<0.01 ;{ }^{* * * *} \mathrm{P}<0.001$ vs. untreated group. COR, cordycepin; PI, propidium iodide.

A

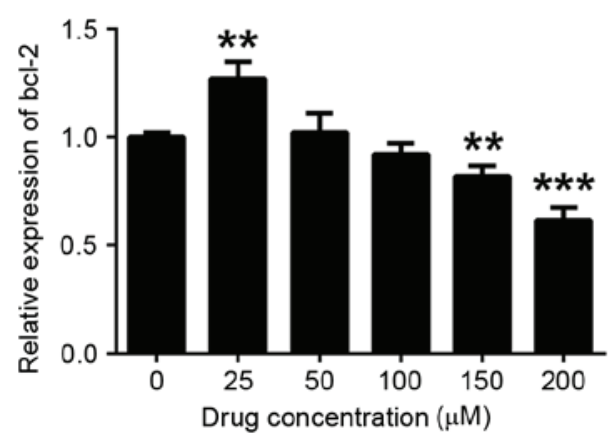

B

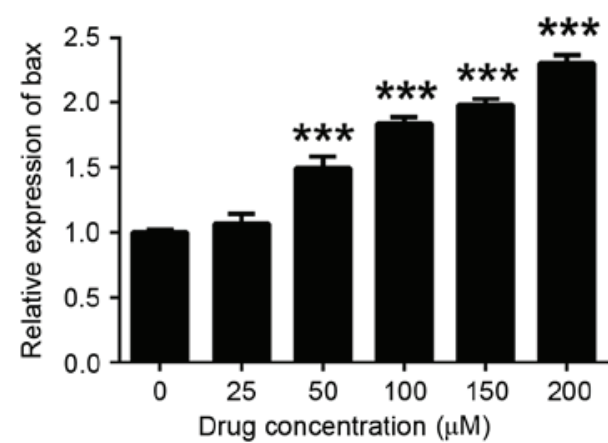

Figure 3. Apoptosis-associated gene expression in THP-1 cells after COR treatment. Reverse-transcription quantitative polymerase chain reaction analysis was employed to detect the expression of (A) Bcl-2 and (B) Bax in COR-treated THP-1 cells. Values are expressed as the mean \pm standard error. ${ }^{* *} \mathrm{P}<0.01 ;{ }^{* * * *} \mathrm{P}<0.001$ vs. untreated group. COR, cordycepin; Bcl-2, B-cell lymphoma 2; Bax, Bcl-2-associated X protein.

aim of the present study was to examine whether COR had anti-cancer activity against AMoL. The results demonstrated that COR exerted a marked inhibitory effect on the THP-1 $\mathrm{AMoL}$ cell line in vitro. COR inhibited the spontaneous growth of THP-1 cells in a dose- and time-dependent manner. To further investigate the mechanisms of the anti-AMoL activity of COR, the effects of COR on apoptosis and activation of Akt/ERK survival signaling as well as Bcl-2/Bax/caspase-3 


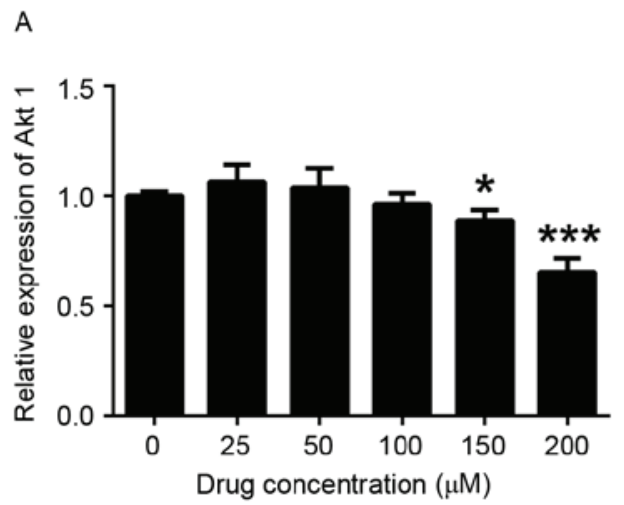

B

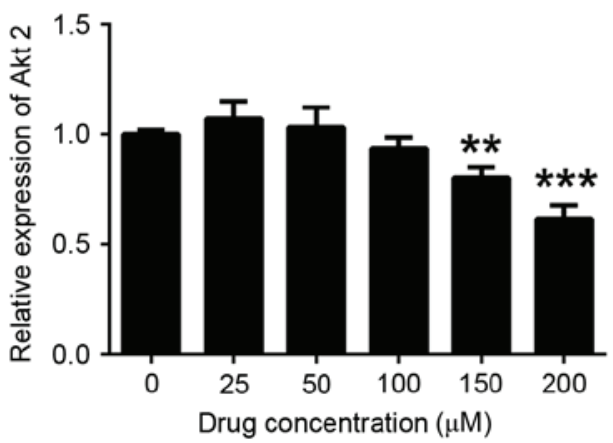

C

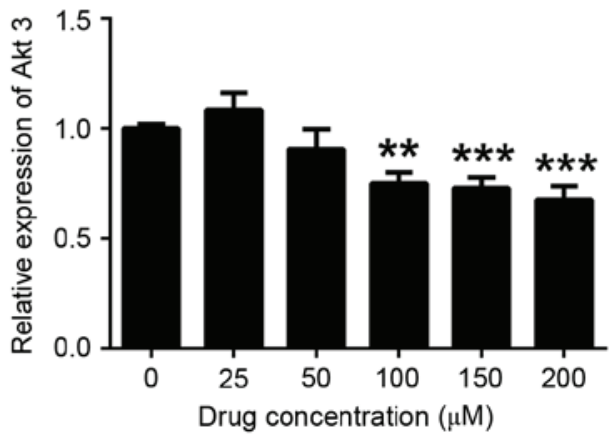

Figure 4. Cell survival-associated gene expression in THP-1 cells after COR treatment. Reverse-transcription quantitative polymerase chain reaction analysis was employed to detect the expression of (A) Akt1, (B) Akt2 and (C) Akt3 in COR-treated THP-1 cells. Values are expressed as the mean \pm standard error. ${ }^{*} \mathrm{P}<0.05 ;{ }^{* *} \mathrm{P}<0.01 ;{ }^{* * *} \mathrm{P}<0.001$ vs. untreated group. COR, cordycepin.

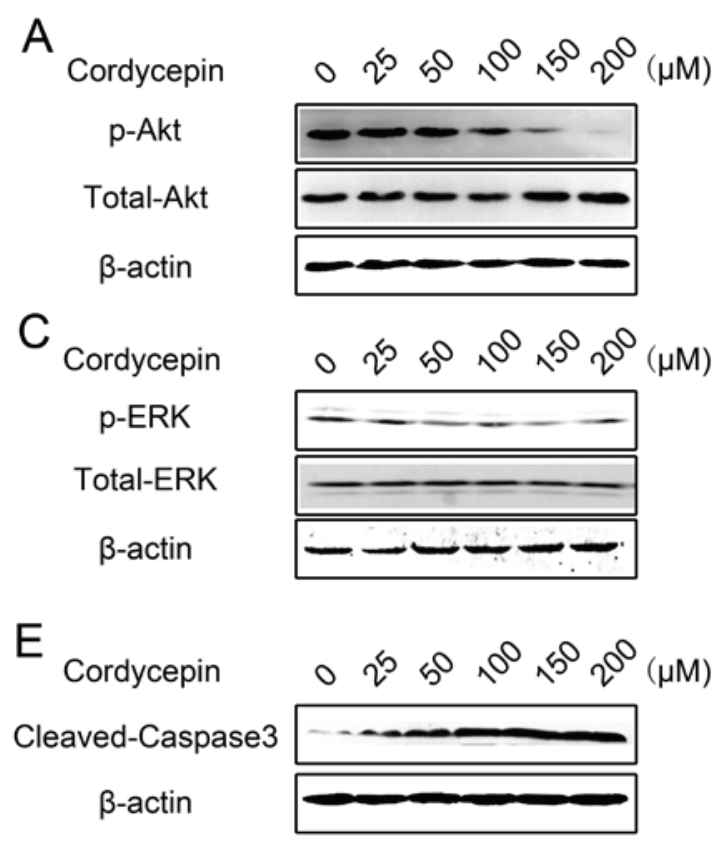

B
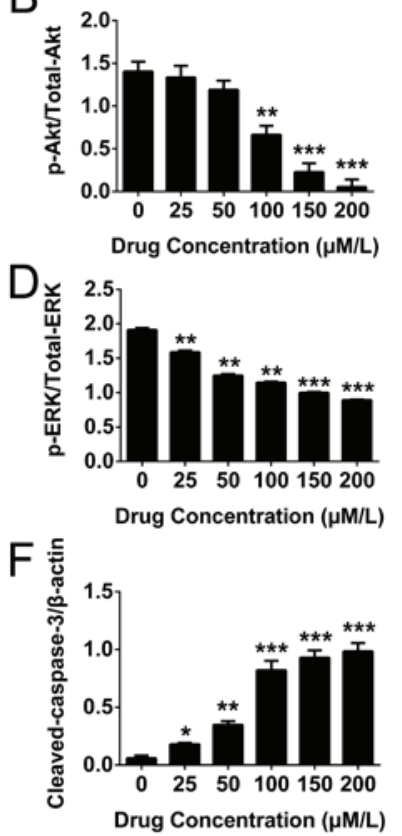

Figure 5. Western blot analysis of p/total-Akt, p-ERK/total-ERK and cleaved caspase-3 levels following apoptosis induction by COR in THP-1 cells. (A-C) Representative western blot displaying the levels of (A) p/total-Akt, (B) quantified, (C) p-ERK/total-ERK, (D) quantifies, (E) cleaved caspase-3 and, (F) quantified in THP-1 cells following COR treatment. Values were normalized to $\beta$-actin. Values are expressed as the mean \pm standard error. ${ }^{*} \mathrm{P}<0.05$; ${ }^{* *} \mathrm{P}<0.01 ;{ }^{* * *} \mathrm{P}<0.001$ vs. untreated group. COR, cordycepin; $\mathrm{p}$-ERK, phosphorylated extracellular signal-regulated kinase.

apoptotic pathways in THP-1 cells were assessed. It was revealed that COR induced apoptosis by inhibiting Akt/ERK signaling and activating caspase-3 following disturbance of the balance of the Bcl-2/Bax axis. 
Leukemia cells often display continued activation of survival and signaling pathways, such as Akt and ERK, due to gene mutations, including rearrangements and chromosome translocation, and survival signaling pathways have an important role in the proliferation, tumorigenesis, evasion of apoptosis and drug resistance (33-36). The more survival signaling pathways are constitutively active in AML, the poorer the prognosis. The results of the present study demonstrated that in the THP-1 AMoL cell line, Akt and ERK signal transduction pathways were simultaneously suppressed. The apoptotic protein caspase-3 was activated by downregulation of the $\mathrm{Bcl}-2 / \mathrm{Bax}$ ratio after $\mathrm{COR}$ treatment. It was speculated that inhibition of the activation of the Akt/ERK pathways and promotion of the activation of caspase- 3 by COR may produce an enhanced response in anti-AMoL therapy. Compared with numerous specific inhibitors, which have a single target for the treatment of leukemia, COR may be more effective, as drug resistance frequently emerges due to hyperactivation of alternative signaling pathways under the treatment with a drug that has a single target. As Akt, ERK and caspase-3 signaling are all interconnected and are not separate pathway entities (37) and COR exhibited characteristics of a multi-targeted therapeutic, it may be deduced that it rarely causes resistance in AMoL, that its efficacy may be higher and that the response duration may be longer.

Based on the above, the role of COR in restricting the proliferation and inducing apoptosis of $\mathrm{AMoL}$ cells was indicated. COR was found to indirectly or directly affect the viability of AMoL cells via Akt/ERK survival signaling and caspase-associated apoptosis signaling, which are key controllers of cell survival and apoptosis (38-41). However, further research should be performed based on this preliminary research. First, the in-depth molecular mechanisms underlying the COR-mediated inhibition of cell survival and induction of apoptosis-associated signaling pathways should be elucidated. Furthermore, animal experiments should be performed to verify the therapeutic effect of COR in vivo. Finally, the efficacy of COR in patients may be assessed.

In conclusion, the present study revealed that COR was efficacious against AMoL in vitro. Furthermore, COR treatment led to a decrease of p-Akt and p-ERK, while increasing the levels of cleaved caspase-3 via increasing the $\mathrm{Bcl}-2 / \mathrm{Bax}$ ratio. These are likely to be downstream mechanisms, by which COR exerts its inhibitory effects in AMoL. However, further study is required to fully elucidate the underlying mechanisms.

\section{Acknowledgements}

This study was supported by grants from the National Natural Science Foundation of China (nos. 81500392 and 31201010), the Shanghai Committee of Science and Technology of China (nos. 12ZR1419500 and 114119a8700) and the Health Bureau of Shanghai (no. ZYSNXD-CCZDYJ029).

\section{References}

1. Li S, Mason C and Melnick A: Genetic and epigenetic heterogeneity in acute myeloid leukemia. Curr Opin Genet Dev 36: 100-106, 2016.

2. Yeh CH,Moles R and Nicot C: Clinical significance of microRNAs in chronic and acute human leukemia. Mol Cancer 15: 37, 2016.
3. Khaled S, Al Malki M and Marcucci G: Acute myeloid leukemia: Biologic, prognostic, and therapeutic insights. Oncology (Williston Park) 30: 318-329, 2016

4. Pettersson L, Levéen P, Axler O, Dvorakova D, Juliusson G and Ehinger M: Improved minimal residual disease detection by targeted quantitative polymerase chain reaction in Nucleophosmin 1 type a mutated acute myeloid leukemia. Genes Chromosomes Cancer 55: 750-766, 2016.

5. Brumatti G, Ma C, Lalaoui N, Nguyen NY, Navarro M, Tanzer MC, Richmond J, Ghisi M, Salmon JM, Silke N, et al: The caspase- 8 inhibitor emricasan combines with the SMAC mimetic birinapant to induce necroptosis and treat acute myeloid leukemia. Sci Transl Med 8: 339ra69, 2016.

6. Yadav M, Singh AK, Kumar H, Rao G, Chakravarti B, Gurjar A, DograS, KushwahaS, Vishwakarma AL, Yadav PN, et al: Epidermal growth factor receptor inhibitor cancer drug gefitinib modulates cell growth and differentiation of acute myeloid leukemia cells via histamine receptors. Biochim Biophys Acta 1860: 2178-2190, 2016.

7. Hu S, Ueda M, Stetson L, Ignatz-Hoover J, Moreton S, Chakrabarti A, Xia Z, Karan G, de Lima M, Agrawal MK and Wald DN: A novel glycogen synthase kinase-3 inhibitor optimized for acute myeloid leukemia differentiation activity. Mol Cancer Ther 15: 1485-1494, 2016.

8. Jackson RC and Radivoyevitch T: Evolutionary dynamics of chronic myeloid leukemia progression: The progressioninhibitory effect of imatinib. AAPS J 18: 914-922, 2016.

9. Foucar K and Anastasi J: Acute myeloid leukemia with recurrent cytogenetic abnormalities. Am J Clin Pathol 144: 6-18, 2015.

10. Banerji L and Sattler M: Targeting mutated tyrosine kinases in the therapy of myeloid leukaemias. Exp Opin Ther Targets 8: 221-239, 2004.

11. Mo J, Lampkin B, Perentesis J, Poole L and Bao L: Translocation $(8 ; 18 ; 16)(\mathrm{p} 11 ; \mathrm{q} 21 ; \mathrm{p} 13)$. A new variant of $\mathrm{t}(8 ; 16)(\mathrm{p} 11 ; \mathrm{p} 13)$ in acute monoblastic leukemia: Case report and review of the literature. Cancer Genet Cytogenet 165: 75-78, 2006.

12. Prayongratana K, Kulpraneet $M, P a n i c h c h o b ~ P$ and Tantisiriwat $W$ : Acute monoblastic leukemia with $\mathrm{t}(10 ; 11)(\mathrm{p} 12 ; \mathrm{q} 23)$ presenting with pulmonary involvement: A case report and literature review. J Med Assoc Thai 91: 559-563, 2008

13. Douet-Guilbert N, Morel F, Le Bris MJ, Sassolas B, Giroux JD and De Braekeleer M: Rearrangement of MLL in a patient with congenital acute monoblastic leukemia and granulocytic sarcoma associated with a $\mathrm{t}(1 ; 11)(\mathrm{p} 36 ; \mathrm{q} 23)$ translocation. Leuk Lymphoma 46: 143-146, 2005.

14. Tasaka T, Matsuhashi Y, Uehara E, Tamura T, Kakazu N, Abe T and Nagai M: Secondary acute monocytic leukemia with a translocation $\mathrm{t}(8 ; 16)(\mathrm{p} 11 ; \mathrm{p} 13)$ : Case report and review of the literature. Leuk Lymphoma 45: 621-625, 2004.

15. Goemans BF, Zwaan CM, Harlow A, Loonen AH, Gibson BE, Hählen K, Reinhardt D, Creutzig U, Heinrich MC and Kaspers GJ: In vitro profiling of the sensitivity of pediatric leukemia cells to tipifarnib: Identification of T-cell ALL and FAB M5 AML as the most sensitive subsets. Blood 106: 3532-3537, 2005.

16. Riccioni R, Senese M, Diverio D, Riti V, Buffolino S, Mariani G, Boe A,Cedrone M,Lo-Coco F,Foà R, etal: M4 and M5 acutemyeloid leukaemias display a high sensitivity to Bortezomib-mediated apoptosis. Br J Haematol 139: 194-205, 2007.

17. Zhang HW, Lin ZX, Tung YS, Kwan TH, Mok CK, Leung $\mathrm{C}$ and Chan LS: Cordyceps sinensis (a traditional Chinese medicine) for treating chronic kidney disease. Cochrane Database Syst Rev 18: Cd008353, 2014.

18. Nakamura K, Shinozuka K and Yoshikawa N: Anticancer and antimetastatic effects of cordycepin, an active component of Cordyceps sinensis. J Pharmacol Sci 127: 53-56, 2015.

19. Tuli HS, Sharma AK, Sandhu SS and Kashyap D: Cordycepin: A bioactive metabolite with therapeutic potential. Life Sci 93: 863-869, 2013.

20. Nakamura K, Konoha K, Yoshikawa N, Yamaguchi Y, Kagota S, Shinozuka K and Kunitomo M: Effect of cordycepin (3'-deoxyadenosine) on hematogenic lung metastatic model mice. In vivo 19: 137-141, 2005.

21. Li Y, Li K, Mao L, Han X, Zhang K, Zhao C and Zhao J: Cordycepin inhibits LPS-induced inflammatory and matrix degradation in the intervertebral disc. Peer J 4: e1992, 2016.

22. Smith MP, Young H, Hurlstone A and Wellbrock C: Differentiation of THP1 cells into macrophages for transwell co-culture assay with melanoma cells. Bio Protoc 5: pii: e1638, 2015.

23. Livak KJ and Schmittgen TD: Analysis of relative gene expression data using real-time quantitative PCR and the 2(-Delta Delta C(T)) Method. Methods 25: 402-408, 2001. 
24. Bertacchini J, Heidari N, Mediani L, Capitani S, Shahjahani M, Ahmadzadeh A and Saki N: Targeting PI3K/AKT/mTOR network for treatment of leukemia. Cell Mol Life Sci 72: 2337-2347, 2015.

25. Barrett D, Brown VI, Grupp SA and Teachey DT: Targeting the $\mathrm{PI} 3 \mathrm{~K} / \mathrm{AKT} / \mathrm{mTOR}$ signaling axis in children with hematologic malignancies. Paediatr Drugs 14: 299-316, 2012.

26. Shafer D and Grant S: Update on rational targeted therapy in AML. Blood Rev 30: 275-283, 2016.

27. Saultz JN and Garzon R: Acute myeloid leukemia: A concise review. J Clin Med 5: pii: E33, 2016

28. Wouters BJ and Delwel R: Epigenetics and approaches to targeted epigenetic therapy in acute myeloid leukemia. Blood 127: 42-52, 2016.

29. Zhang P, Huang C, Fu C, Tian Y, Hu Y, Wang B, Strasner A, Song Y and Song E: Cordycepin (3'-deoxyadenosine) suppressed HMGA2, Twist1 and ZEB1-dependent melanoma invasion and metastasis by targeting miR-33b. Oncotarget 6 : 9834-9853, 2015.

30. Yamamoto K, Shichiri H, Uda A, Yamashita K, Nishioka T, Kume M, Makimoto H, Nakagawa T, Hirano T and Hirai M Apoptotic effects of the extracts of cordyceps militaris via Erk phosphorylation in a renal cell carcinoma cell line. Phytother Res 29: 707-713, 2015.

31. Yang X, Li Y, He Y, Li T, Wang W, Zhang J, Wei J, Deng Y and Lin R: Cordycepin alleviates airway hyperreactivity in a murine model of asthma by attenuating the inflammatory process. Int Immunopharmacol 26: 401-408, 2015.

32. Ramesh T, Yoo SK, Kim SW, Hwang SY, Sohn SH, Kim IW and Kim SK: Cordycepin (3'-deoxyadenosine) attenuates age-related oxidative stress and ameliorates antioxidant capacity in rats. Exp Gerontol 47: 979-987, 2012.

33. Shao X, Liu Y, Li Y, Xian M, Zhou Q, Yang B, Ying M and He Q: The HER 2 inhibitor TAK165 sensitizes human acute myeloid leukemia cells to retinoic acid-induced myeloid differentiation by activating MEK/ERK mediated RAR $\alpha /$ STAT1 axis. Sci Rep 6: 24589, 2016.
34. Bi L, Wu J, Ye A, Wu J, Yu K, Zhang S and Han Y: Increased Th17 cells and IL-17A exist in patients with B cell acute lymphoblastic leukemia and promote proliferation and resistance to daunorubicin through activation of Akt signaling. J Transl Med 14: 132, 2016.

35. Waibel M, Gregory G, Shortt J and Johnstone RW: Rational combination therapies targeting survival signaling in aggressive B-cell leukemia/lymphoma. Curr Opin Hematol 21: 297-308, 2014.

36. Guo Y, Shan Q, Gong Y, Lin J, Shi F, Shi R and Yang X: Curcumin induces apoptosis via simultaneously targeting AKT/mTOR and RAF/MEK/ERK survival signaling pathways in human leukemia THP-1 cells. Pharmazie 69: 229-233, 2014.

37. Zhang S, He Y, Tong Q, Chen Q, Wu X and Huang W: Deltonin induces apoptosis in MDA-MB-231 human breast cancer cells via reactive oxygen species-mediated mitochondrial dysfunction and ERK/AKT signaling pathways. Mol Med Rep 7: 1038-1044, 2013.

38. Reynolds C, Roderick JE, LaBelle JL, Bird G, Mathieu R, Bodaar K, Colon D, Pyati U, Stevenson KE, Qi J, et al: Repression of BIM mediates survival signaling by MYC and AKT in high-risk T-cell acute lymphoblastic leukemia. Leukemia 28: 1819-1827, 2014

39. Zhu GH, Dai HP, Shen Q, Ji O, Zhang Q and Zhai YL: Curcumin induces apoptosis and suppresses invasion through MAPK and MMP signaling in human monocytic leukemia SHI-1 cells. Pharm Biol 54: 1303-11, 2016.

40. Wang $X$, Pesakhov S, Harrison JS, Danilenko $M$ and Studzinski GP: ERK5 pathway regulates transcription factors important for monocytic differentiation of human myeloid leukemia cells. J Cell Physiol 229: 856-867, 2014.

41. Gonnella R, Santarelli R, Farina A, Granato M, D'Orazi G, Faggioni A and Cirone M: Kaposi sarcoma associated herpesvirus (KSHV) induces AKT hyperphosphorylation, bortezomib-resistance and GLUT-1 plasma membrane exposure in THP-1 monocytic cell line. J Exp Clin Cancer Res 32: 79, 2013. 